EPJ Web of Conferences 73, 02012 (2014)

DOI: $10.1051 /$ epjconf/20147302012

(C) Owned by the authors, published by EDP Sciences, 2014

\title{
Spin studies via Drell-Yan processes at PANDA
}

\author{
M. Destefanis ${ }^{\mathrm{a}}$ on behalf of the PANDA Collaboration \\ Università degli Studi di Torino and INFN Sezione di Torino, Italy
}

\begin{abstract}
The nucleon structure is still not completely understood. A transverse momentum dependent description of the nucleon structure is a crucial milestone for several forthcoming studies in a wide range of experimental scenarios. By means of antiproton beams, possibly polarized in a later stage of the project, with a beam momentum up to $15 \mathrm{GeV} / \mathrm{c}$, which will be available at the future FAIR facility, the nonperturbative region of QCD is planned to be investigated. One of the main goals of the forthcoming experiments at FAIR is the study of Drell-Yan lepton pairs by means of proton-antiproton annihilations, taking also advantage of the expected high luminosity. The Drell-Yan production is a unique tool to access the spin dependent properties of the nucleon, and in particular its transverse degrees of freedom. Transverse Momentum Dependent (TMD) Parton Distribution Functions (PDFs), i.e. the Boer-Mulders function, the Sivers function, and the Transversity, could be deeply investigated by means of experimental angular asymmetries. In later stages of FAIR, single- and double-spin asymmetries could be investigated as well. The Drell-Yan physics program which could be accessed at FAIR with the PANDA experiment will be discussed in details, in the light of existing results in the field.
\end{abstract}

\section{Introduction}

The nucleon structure can be described completely by the Parton Distribution Functions (PDF) and by the Fragmentation Functions (FF). At leading twist, the unpolarized data can be explained by three PDFs $\left(f_{1}(x), g_{1}(x)\right.$, and $\left.h_{1}(x)\right)$, which depict the quark structure of a hadron. $f_{1}(x)$ describes the probability of finding a quark with a fraction $x$ of the longitudinal momentum of the parent hadron regardless on the quark's spin orientation; $g_{1}(x)$ is the quarks' helicity distribution in a longitudinally polarized hadron; $h_{1}(x)$, the so called Transversity function, depicts the quark's transverse spin distribution inside a transversely polarized hadron. The first two PDFs have a probabilistic interpretation in the helicity base and many experimental data, characterized by a high accuracy, can be found in the litterature; the $h_{1}(x)$ function is not diagonal in the helicity base, and it is still subject of deep investigations. The Transversity is a chirally odd function, and, for this reason, it cannot be extracted from the historical deep inelastic scattering (DIS) data. The Soffer inequality

$$
\left|h_{1}(x)\right| \leq \frac{1}{2}\left|f_{1}(x)+g_{1}(x)\right|
$$

\footnotetext{
${ }^{a}$ e-mail: marco.destefanis@to.infn.it
}

This is an Open Access article distributed under the terms of the Creative Commons Attribution License 4.0, which permits unrestricted use, distribution, and reproduction in any medium, provided the original work is properly cited. 


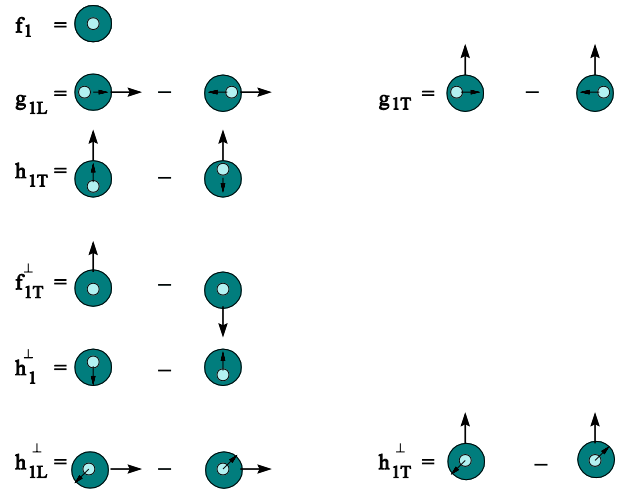

Figure 1. TMD PDF's distributions. Arrows indicate the polarizations of the quark and of the parent hadron.

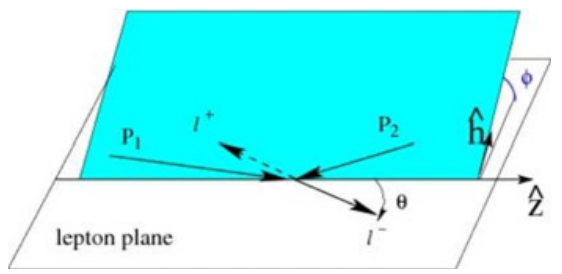

Figure 2. The Collins-Soper frame [2].

which has some limitations, but holds at NLO while considering a large scale, connects the three mentioned distribution functions.

The polarized cross sections in high energy hadron-hadron scattering [1] can however be described in a consistent way only if a Transverse Momentum Dependence (TMD) approach is introduced. This approach takes into account the transverse momentum of the partons $\left(\mathbf{k}_{T}\right)$, and needs eight independent PDFs, which are functions of $x$ and $\mathbf{k}_{T}$. According to their chirality, those TMD PDFs, shown in Fig. 1 can be divided into chirally even $\left(f_{1}, g_{1 L}, f_{1 T}^{\perp}\right.$, and $\left.g_{1 T}\right)$ and chirally odd $\left(h_{1}^{\perp}, h_{1 L}^{\perp}, h_{1 T}\right.$, and $\left.h_{1 T}^{\perp}\right)$ functions.

\section{Experimental scenarios}

The TMD PDFs can be investigated in different experimental scenarios: Semi-Inclusive Deep Inelastic Scattering (SIDIS) and Drell-Yan (DY) processes. In the first case, the TMD PDFs are convoluted with the fragmentation functions (FF) and their extraction poses both theoretical and experimental challenges. The latter scenario allows a direct access to those functions, since it is possible to define experimental azimuthal asymmetries depending on the TMD PDFs only. The low center of mass energy range which should be available at FAIR will grant the access to a unique kinematic region where valence quark effects dominate. In addition, in the $p \bar{p}$ annihilation reaction each valence quark can contribute to the DY diagram. The DY is an electromagnetic process in which a quark and an antiquark annihilate into a virtual photon, which then decays into a lepton pair final state $\left(h_{1} h_{2} \rightarrow \gamma^{*} X \rightarrow l^{+} l^{-} X\right)$. The rest frame of the virtual photon, the so called Collins-Soper frame [2], is usually introduced to describe DY events. As shown in Fig. 2, in this frame a hadron and a lepton planes can be defined, $\varphi$ is the angle between those planes, and $\varphi_{S_{1,2}}$ is the angle between the nucleon spin $\left(\mathrm{S}_{1,2}\right)$ and the lepton plane. 


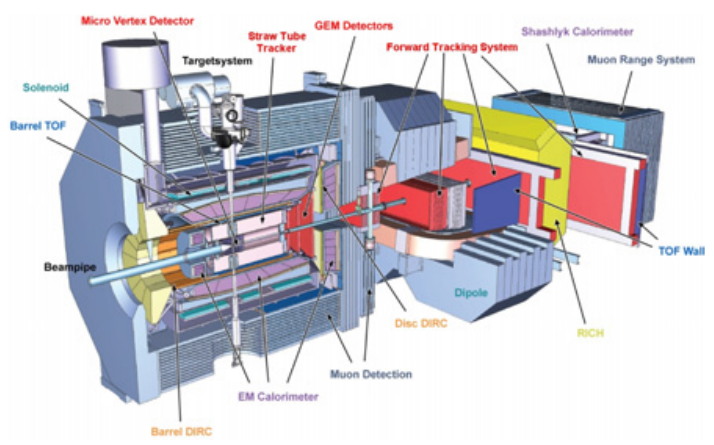

Figure 3. 3D view of the PANDA spectrometer.

In the completely unpolarized case, a $\cos 2 \varphi$ contribution to the DY differential cross section [3] provides asymmetries which could lead to the product $\bar{h}_{1}^{\perp} h_{1}^{\perp} . h_{1}^{\perp}$, the so called Boer-Mulders function, is a T-odd chirally odd TMD function which describes the distribution of transversely polarized quarks inside an unpolarized nucleon (see Fig. 1). In the single polarized case, the DY differential cross section includes further terms. Those terms depend on $\varphi_{S}$ and lead to single spin asymmetries weighted by $\sin \left(\varphi-\varphi_{S_{2}}\right)$ and by $\sin \left(\varphi+\varphi_{S_{2}}\right)$ and proportional to four of the TMD PDFs discussed above: $h_{1}^{\perp}$ and Transversity, and $f_{1}$ and $f_{1 T}^{\perp}$ [3]. $f_{1 T}^{\perp}$ is the so called Sivers function and describes the unpolarized quark distribution regardless of the spin orientation inside a transversely polarized hadron.

\section{Drell-Yan processes at PANDA}

The future FAIR facility is designed to take advantage of different storage rings: among them, the High Energy Storage Ring (HESR) will be devoted to the antiproton accumulation and acceleration. The HESR will be equipped with stochastic and electron cooling systems in order to achieve the required luminosity. PANDA [4] will be a fixed target experiment hosted in the HESR storage ring. The PANDA detector, presented in Fig. 3, is designed to cover almost the full solid angle acceptance. Its modular design foresees two magnetic spectrometers, the first one surrounds the interaction point and it is characterized by a superconducting solenoid, and the second one, equipped with a dipole magnet, is dedicated to the most forward emitted particles.

The investigation of the electromagnetic processes will be one of the main aims of the PANDA physics program. Among the others, the foreseen high luminosity mode (up to $2 \cdot 10^{32} \mathrm{~cm}^{-2} \mathrm{~s}^{-1}$ ) should allow the study of the DY production of muon pairs in processes like $p \bar{p} \rightarrow \gamma^{*} X \rightarrow \mu^{+} \mu^{-} X$. The DY process is usually studied in the safe region $4 \leq \mathrm{M}_{\gamma^{*}} \leq 9 \mathrm{GeV} / \mathrm{c}^{2}$, so called because free from resonances contributions. PANDA is planning to investigate also the $1.5 \leq \mathrm{M}_{\gamma^{*}} \leq 2.5 \mathrm{GeV} / \mathrm{c}^{2}$ region, since free from resonances as well and characterized by an higher DY cross section (about three orders of magnitude).

At the maximum center of mass energy available $\left(s=30 \mathrm{GeV}^{2}\right)$, the DY cross section is estimated to be of the order of $1 \mathrm{nb}$ [4]. The production of events of the type $p \bar{p} \rightarrow n\left(\pi^{+} \pi^{-}\right) X$, where $n$ indicates the number of pion pairs, has been investigated in the PANDA scenario as main background source. The background cross section is expected to be around $20 \div 30 \mathrm{mb}$ [4], leading to a required rejection factor $\sim 10^{7}$.

The generator described in [5] was used to understand the feasibility of the measurement. It can provide final states containing muon pairs produced in $\bar{p}$ annihilations with polarized or unpolarized nuclear targets, and it is based on the experimental data available in the litterature. Figure 4 shows the described asymmetries as a function of the longitudinal momentum of the hadronic probe $\left(x_{p}\right)$ for the unpolarised and the single-polarised cases, reconstructed from $5 \cdot 10^{5}$ simulated events, corresponding to 


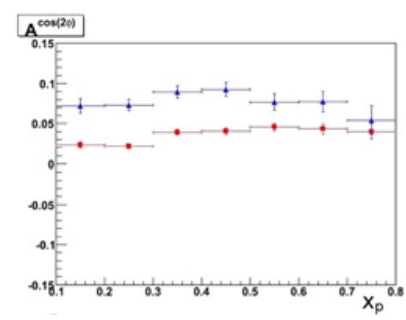

(a)

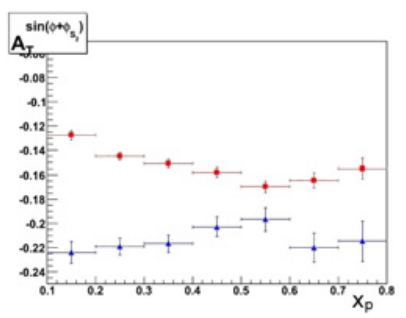

(b)

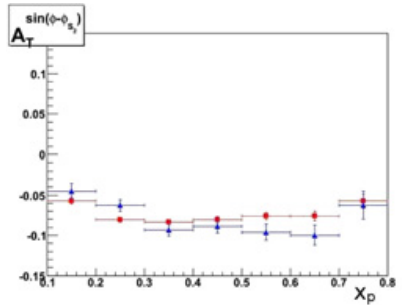

(c)

Figure 4. Simulated experimental asymmetries corresponding to the terms of the differential DY cross section [3] weighted by $\cos 2 \varphi$ (a), $\sin \left(\varphi+\varphi_{S_{2}}\right)$ (b), and $\sin \left(\varphi-\varphi_{S_{2}}\right)$ (c), plotted as a function of $x_{p}$, the longitudinal momentum fraction of the hadronic probe. Statistical error bars correspond to about 5 months data taking at full luminosity.

about 5 months data taking at full luminosity. The $x$ dependence of the asymmetries has been introduced in order to probe the accessible kinematic region only and it is not relevant in these simulations. The azimuthal asymmetries are here presented for two different selected kinematic regions of the transverse momentum of the muon pair $\left(\mathrm{q}_{T}\right): 1 \leq \mathrm{q}_{T} \leq 2 \mathrm{GeV} / \mathrm{c}$ (red squares), and $2 \leq \mathrm{q}_{T} \leq 3 \mathrm{GeV} / \mathrm{c}$ (blue triangles). Acceptance and efficiency corrections, crucial for such kind of measurements, are still under investigation. The small error bars, presented in Fig. 4, indicate the possibility to perform a scan of these asymmetries in different muon pairs' transverse momentum regions. This investigation can be performed in order to probe the limits of the perturbative approach and to balance soft and hard contributions to the DY processes in the PANDA energy range. In the single-polarized case we expect comparable or even larger azimuthal asymmetries with respect to the unpolarized scenario.

Taking into account the geometrical acceptance, the material budget, and the preliminary estimated reconstruction efficiency $(\sim 33 \%)$, at the highest foreseen luminosity $\left(2 \cdot 10^{32} \mathrm{~cm}^{-2} \mathrm{~s}^{-1}\right), 130 \mathrm{~K}$ DY events are expected per month. In such an experimental scenario, one year data taking at the highest beam energy should already allow to determine the azimuthal asymmetries with uncertainties of the order of the presented ones. The optimization of the background rejection is still under investigation.

\section{References}

[1] C. Bourrely and J. Soffer, Eur. Phys. J. C 36, 371 (2004)

[2] J.C. Collins and D.E. Soper, Phys. Rev. D 16, 2219 (1977)

[3] M. Burkardt and D.S. Hwang, Phys. Rev. D 69, 074032 (2004)

[4] W. Erni, et al., arXiv:0903.3905v1 [hep-ex]

[5] A. Bianconi, Nucl. Instrum. Meth. A 593, 562-571 (2008) 